\title{
Prognostic Factors and Long-term Surgical Outcomes for Exudative Age-related Macular Degeneration with Breakthrough Vitreous Hemorrhage
}

\author{
Tae Young Kim ${ }^{1}$, Hyun Goo Kang ${ }^{1,2}$, Eun Young Choi ${ }^{1,2}$, Hyoung Jun $\mathrm{Koh}^{3}$, Sung Soo Kim ${ }^{3}$, Ji Hwan Lee ${ }^{3}$, Min Kim¹, \\ Suk Ho Byeon ${ }^{3}$, Christopher Seungkyu Lee ${ }^{1,3}$ \\ ${ }^{1}$ Department of Ophthalmology, Institute of Vision Research, Gangnam Severance Hospital, Yonsei University College of Medicine, \\ Seoul, Korea \\ ${ }^{2}$ Department of Medicine, Yonsei University Graduate School, Seoul, Korea \\ ${ }^{3}$ Department of Ophthalmology, Institute of Vision Research, Severance Eye Hospital, Yonsei University College of Medicine, Seoul, \\ Korea
}

Purpose: We sought to evaluate the long-term outcomes for patients with exudative age-related macular degeneration (AMD) undergoing vitrectomy for breakthrough vitreous hemorrhage and to investigate possible prognostic factors.

Methods: Consecutive patients treated at two high-volume referral-based tertiary hospitals between July 2006 and December 2019 were retrospectively reviewed. Surgery was performed using the standard three-port vitrectomy. The primary outcome was the change in best-corrected visual acuity (BCVA) over long-term follow-up, while secondary outcomes included the assessment of possible prognostic factors.

Results: Among 50 eyes from 50 patients included in this study, 23 (46\%) were diagnosed with polypoidal choroidal vasculopathy (PCV) and 27 (54\%) were diagnosed with neovascular AMD. Preoperative vision at the time of vitreous hemorrhage onset was 20 / 3,027 (logarithm of the minimum angle of resolution [logMAR], $2.18 \pm 0.34$ ). At 12 months after surgery, the mean BCVA improved to 20 / 873 (logMAR, $1.64 \pm 0.76 ; p<0.001$ ). At 24 months, the BCVA was 20 / 853 (logMAR, $1.63 \pm$ $0.75 ; p<0.001$ ). Univariate analysis revealed that older age (odds ratio [OR], 0.879; $p=0.007$ ] and the presence of submacular hemorrhage (OR, 0.081; $p=0.022)$ were factors associated with a poor 2-year visual outcome. Multivariable regression showed that older age (OR, 0.876; $p=0.026)$ and neovascular AMD (as compared with PCV) $(\mathrm{OR}, 0.137 ; p=0.014)$ were significant negative factors influencing the 2-year visual outcome. The mean injection interval prior to vitrectomy was 4.53 months, which extended to 27.64 months after vitrectomy $(p=0.028)$.

Conclusions: Younger age, the absence of submacular hemorrhage, and PCV type were associated with a favorable 2-year visual outcome after vitrectomy for vitreous hemorrhage in patients with exudative AMD. Overall, vitrectomy resulted in improved visual acuity and patients showed a decreased need for anti-vascular endothelial growth factor therapy thereafter.

Key Words: Macular degeneration, Prognosis, Vitrectomy, Vitreous hemorrhage

Received: February 7, 2020 Final revision: March 20, 2020 Accepted: April 10, 2020

Corresponding Author: Christopher Seungkyu Lee, MD, PhD. Department of Ophthalmology, Institute of Vision Research, Gangnam Severance Hospital, Yonsei University College of Medicine, 211 Eonjuro, Gangnam-gu, Seoul 06273, Korea. Tel: 82-2-2019-3440, Fax: 82-2-3463-1049, E-mail: sklee219@yuhs.ac 
Age-related macular degeneration (AMD) leads to severe vision loss and legal blindness, with a prevalence rate of up to $8.7 \%$ worldwide, and is especially common in developed countries [1,2]. If not treated appropriately, exudative AMD (eAMD) may lead to massive submacular hemorrhages, breakthrough vitreous hemorrhage, disciform scarring, and uncommonly to severe serous retinal detachment, which are all detrimental to long-term vision quality. Vitreous hemorrhage is a rare complication of eAMD, occurring reportedly at a rate of $2 \%$ to $7 \%$, but has a very critical impact on the visual prognosis [3]. Previous studies on surgical outcomes and prognostic factors for vitreous hemorrhage associated with eAMD have reported conflicting results. Some studies have suggested that severe inflammatory reactions in the postoperative period after vitrectomy may explain the poor visual outcomes, with aggravation of the underlying inflammatory process inherent in eAMD. Others have reported that surgical intervention appears to result in favorable visual outcomes but did not discuss any prognostic factors [4-6].

Our understanding is further complicated by the mix of eAMD with polypoidal choroidal vasculopathy (PCV) in past studies, as PCV may be a pachychoroid-driven disorder different from that of typical neovascular AMD. Although a few recent reports have focused solely on the results of treatment for PCV with breakthrough vitreous hemorrhage and evaluated possible predictive factors, no comparative analysis with long-term outcomes have been performed $[7,8]$. Thus, the current literature is sparse regarding the long-term (over 2 years) visual and surgical outcomes of eAMD with vitreous hemorrhage and prognostic factors that may aid clinicians in guiding patients' expectations. There are also conflicting views pertaining to the safety of performing combined cataract surgery with vitrectomy for eAMD with vitreous hemorrhage due to the possible aggravation of inflammation.

In this study, we investigated and compared the long-term visual and surgical outcomes for patients with either neovascular AMD or PCV undergoing vitrectomy for breakthrough vitreous hemorrhage. We further evaluated possible prognostic factors influencing long-term outcomes and assessed the safety of combining cataract surgery with vitrectomy.

\section{Materials and Methods}

This was a retrospective case series of consecutive patients diagnosed with vitreous hemorrhage associated with either neovascular AMD or PCV undergoing vitrectomy from July 2006 to December 2019 at two high-volume, referral-based tertiary hospitals, Severance Eye Hospital and Gangnam Severance Hospital, affiliated with Yonsei University College of Medicine. Institutional review board/ ethics committee approval was obtained from Gangnam Severance Hospital (3-2019-0378) and the study adhered to the tenets of the Declaration of Helsinki. Patient consent was waived after institutional review board review as this study was conducted retrospectively with no identifying features of individual patients.

Multimodal imaging was conducted to determine the underlying cause for vitreous hemorrhage, including ultra-widefield fundus photography, optical coherence tomography (OCT), fluorescein angiography, and indocyanine green angiography with a confocal laser-scanning system (HRA-2; Heidelberg Engineering, Heidelberg, Germany). PCV was diagnosed if indocyanine green angiography showed polypoidal structures along with branching vascular networks or OCT revealed typical features. Patients not showing features of PCV and displaying findings of type 1 and 2 choroidal neovascularization or retinal angiomatous proliferation (type 3 choroidal neovascularization) were all included as cases of neovascular AMD. Massive submacular hemorrhage was defined as hemorrhage greater than 4-disc diameters in the greatest linear length, with the determination made by two graders (TYK and HGK); when these graders disagreed, a final determination was made by the supervising grader (CSL).

Patients who were followed up with for a minimum of 12 months after surgery were selected to undergo the analysis of the long-term outcomes. Patients with eyes with vitreous hemorrhage due to proliferative diabetic retinopathy or retinal vein occlusion and those having other ophthalmologic diseases such as macular hole, choroidal melanoma, and rhegmatogenous retinal detachment were excluded from this research.

Surgery was performed by experienced retinal specialists (HJK, JHL, MK, CSL, SHB, and SSK). The standard three-port vitrectomy with either the 23- or 25-gauge system was used according to each surgeon's preference. Adjuvant surgical techniques such as combined cataract sur- 
gery, intravitreal anti-vascular endothelial growth factor (VEGF), intravitreal tissue plasminogen activator (tPA), gas (SF6 or C3F8)/silicone oil tamponade or drainage of submacular hemorrhage were performed at the discretion of the attending surgeon. Changes in preoperative and postoperative anti-VEGF injection frequency and numbers were assessed together with the mean duration interval between injections. Patients who received continuous anti-VEGF injections with at least four injections prior to surgery were included for subgroup analysis. We defined the injection interval as the average injection time between two consecutive intravitreal anti-VEGF injections.

Visual acuity (VA) was measured by Snellen chart and converted to logarithm of the minimum angle of resolution ( $\log$ MAR); furthermore, counting fingers was graded as 20 / 2,000 (logMAR 2.0), hand motion was graded as 20 / 3,990 (logMAR 2.3), light perception was graded as logMAR 2.6, and no light perception was graded as logMAR 2.90. Vision was recorded for up to 2 years of follow-up to analyze the visual change throughout the treatment duration. To analyze prognostic factors, vision better than counting fingers was defined as "favorable VA" $[9,10]$. We defined VA at the time of vitreous hemorrhage as preoperative VA, while initial VA was recorded when patients first visited the clinic.

Finally, we analyzed changes in OCT features including

Table 1. Baseline characteristics $(n=50)$

\begin{tabular}{|c|c|}
\hline Characteristics & Value \\
\hline Age (yr) & $71.4 \pm 8.1(52-83)$ \\
\hline \multicolumn{2}{|l|}{ Sex } \\
\hline Male & $27(54.0)$ \\
\hline Female & $23(46.0)$ \\
\hline Hypertension & $24(58.0)$ \\
\hline Diabetes & $9(18.0)$ \\
\hline Pseudophakic eyes & $4(8.0)$ \\
\hline Presence of submacular hemorrhage & $39(78.0)$ \\
\hline \multicolumn{2}{|l|}{ Visual acuity (logMAR, Snellen equivalent) } \\
\hline At the time of vitreous hemorrhage & $2.18 \pm 0.34(20 / 3,027)^{*}$ \\
\hline Last follow-up & $1.67 \pm 0.76(20 / 935)$ \\
\hline \multicolumn{2}{|l|}{ Wet AMD type } \\
\hline $\mathrm{PCV}$ & $23(46.0)$ \\
\hline Neovascular AMD (types 1 and 2) & $26(52.0)$ \\
\hline RAP (type 3) & $1(2.0)$ \\
\hline Mean postoperative follow-up (mon) & $53.25 \pm 40.39$ \\
\hline \multicolumn{2}{|l|}{ Combined surgery } \\
\hline Anti-VEGF & $28(56.0)$ \\
\hline Anti-VEGF + gas tamponade + tPA & $3(6.0)$ \\
\hline Gas tamponade & $2(4.0)$ \\
\hline Oil tamponade & $5(10.0)$ \\
\hline Oil tamponade + tPA & $1(2.0)$ \\
\hline Oil tamponade + submacular hemorrhage drainage & $1(2.0)$ \\
\hline Cataract surgery & $42(84.0)$ \\
\hline
\end{tabular}

Values are presented as mean \pm standard deviation or number $(\%)$.

$\operatorname{logMAR}=$ logarithm of the minimal angle of resolution; AMD = age-related macular degeneration; PCV = polypoidal choroidal vasculopathy; RAP = retinal angiomatous proliferation; VEGF = vascular endothelial growth factor; tPA = tissue plasminogen activator.

${ }^{*}$ Finger counting $=2.00 \operatorname{logMAR}$, hand motion $=2.30 \operatorname{logMAR}$, light perception $=2.60 \log \mathrm{MAR}$, no light perception $=2.90$. 
central foveal thickness (CFT), the presence of subretinal hemorrhages, and the appearance of subfoveal disciform scars at preoperation and at 1 and 24 months after the operation. We defined CFT as the distance between the internal limiting membrane and Brunch's membrane at the fovea center and measured it by using the tool provided in the OCT software.

Data were analyzed using IBM SPSS Statistics ver. 21.0 (IBM Corp., Armonk, NY, USA). Variables including VA and injection parameters between groups were compared using two-sample paired $t$-tests. Logistic binary regression analysis was used to determine the predictive factors for visual outcomes. A $p$-value of $<0.05$ was considered to be statistically significant.

\section{Results}

Fifty eyes from 50 patients who met the inclusion criteria from a review of 382 patients were included; of these, 23 (46\%) were diagnosed with PCV and 27 (54\%) were diagnosed with neovascular AMD, including one patient who had retinal angiomatous proliferation. The baseline characteristics are summarized in Table 1 . The mean age was 71 years (range, 52-83 years), with no definite predilection for either sex. The mean initial VA was $20 / 873$ ( $\log$ MAR, $1.64 \pm 0.73)$. Breakthrough vitreous hemorrhage was present at the first visit in 24 patients. In the remaining 26 patients, mean VA before the onset of vitreous hemorrhage was 20/408 (logMAR, $1.31 \pm 0.71)$. Preoperative VA measured at the time of vitreous hemorrhage was 20/3027 ( $\log$ MAR, $2.18 \pm 0.34)$. The mean duration of follow-up after surgery was 53.3 months (median, 36 months; range, 12-169 months), while the mean preoperative follow-up period was 12.3 months (median, 3 months; range, 0-104 months). Combined cataract surgery was performed in 42 eyes (84\%). Silicone oil tamponade was performed in seven eyes, while gas tamponade was performed in five eyes (including four eyes with C3F8 and one with SF6). Intravitreal tPA was deployed in four eyes $(8 \%)$ and intravitreal anti-VEGF was administered in 31 eyes $(62 \%)$ during surgery. The injection interval prior to surgery was $3.31 \pm$ 3.92 months (median, 1.71 months; range, 0-17.16 months), whereas the postoperative injection interval frequency was $17.24 \pm 30.54$ months (median, 6.50 months; range, 1.54159.00 months).

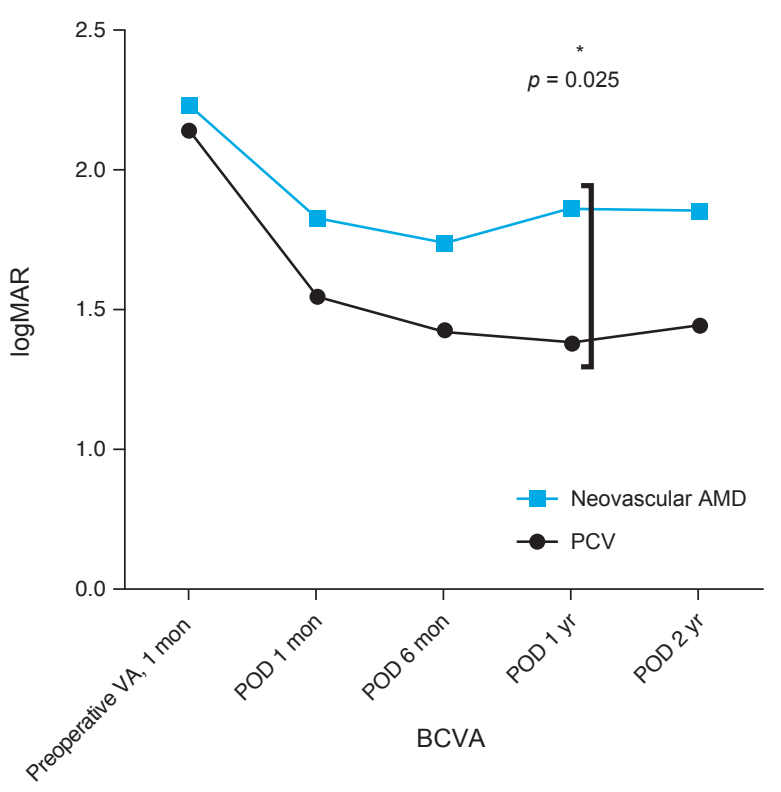

Fig. 1. Displaying preoperative visual acuity (VA) and postoperative VA at 1,6 , and 12 months and 2 years after vitrectomy. Results represent the best-corrected visual acuity (BCVA) converted to logarithm of the minimum angle of resolution $(\log M A R)$. BCVA improvement at different postoperative time points was compared with the baseline preoperative VA and were all statistically significant ( $p<0.001$, two-sample paired $t$-tests). Polypoidal choroidal vasculopathy (PCV) and neovascular age-related macular degeneration (AMD) showed significant BCVA difference at 1 year after vitrectomy only $(p<0.05)$. POD $=$ postoperative day.

\section{Best-corrected VA}

VA improved significantly after vitrectomy (Fig. 1). The overall mean postoperative best-corrected VA (BCVA) improved from $20 / 3,027(\operatorname{logMAR}, 2.18 \pm 0.34)$ to $20 / 873$ (logMAR, $1.64 \pm 0.76 ; p<0.001)$ at 12 months, and to 20 / $853(\log$ MAR, $1.63 \pm 0.75 ; p<0.001)$ at 24 months. The improvement was further maintained until 36 months after surgery $(\mathrm{n}=24, p<0.001)$.

Next, we compared changes in VA based on eAMD subtype, i.e., PCV $(n=23)$ versus neovascular AMD $(n=27)$. Preoperative VA was not different between the PCV and neovascular AMD groups (20 / 2,700 vs. 20 / 3,400, respectively; $p=0.353$ ). While vision at the first postoperative year demonstrated a significant difference between the subtypes (PCV 20 / 480 vs. neovascular AMD 20 / $1,448 ; p=0.025)$, the difference was no longer statistically significant at 2 years (PCV 20 / 563 vs. neovascular AMD $20 / 1,415 ; p=0.055)$ (Fig. 1). 
Table 2. Optical coherence tomography features

\begin{tabular}{lcccc}
\hline & $\begin{array}{c}\text { Preoperative } \\
(\mathrm{n}=21)\end{array}$ & $\begin{array}{c}\text { Postoperative 1 mon } \\
(\mathrm{n}=35)\end{array}$ & $\begin{array}{c}\text { Postoperative 24 mon } \\
(\mathrm{n}=39)\end{array}$ & $\begin{array}{c}p \text {-values for } \\
\text { preoperative vs. postoperative 24 mon } \\
/ / \text { postoperative 1 mon vs. 24 mon }\end{array}$ \\
\hline CFT $(\mu \mathrm{m})$ & $699.8 \pm 356.7$ & $432.1 \pm 282.0$ & $408.9 \pm 271.6$ & $0.036^{*} / / 0.038^{*}$ \\
Subretinal hemorrhage & $21(100)$ & $29(82.9)$ & $16(41.0)$ & $<0.001^{*} / / 0.001^{*}$ \\
Subfoveal disciform scarring & - & $14(40.0)$ & $30(76.9)$ & $\mathrm{NA} / /<0.001^{*}$ \\
\hline
\end{tabular}

Values are presented as mean \pm standard deviation or number (\%); Two-sample paired $t$-test and McNemar's test. $\mathrm{CFT}=$ central foveal thickness; $\mathrm{NA}=$ not applicable. ${ }^{*} p<0.05$.

\section{Anti-VEGF injections}

Changes to the pattern of intravitreal anti-VEGF treatment before and after vitrectomy were analyzed in 11 patients with clinical data prior to surgery. The mean preoperative follow-up and postoperative follow-up periods were 36.82 months and 49.09 months, respectively. The mean injection interval prior to vitrectomy was 4.53 months (median, 3.53 months; range, 1.07-11.15 months); after vitrectomy, the injection interval significantly increased to 27.64 months (median, 10.03 months; range, 1.05-64.14 months; $p=0.028)$. Further, the mean number of intravitreal anti-VEGF injections decreased from $8.64 \pm 4.99$ injections (median, 7 injections) before surgery to $2.64 \pm 2.87$ (median, 2 injections) after surgery in a manner that was also significant $(p<0.001)$.

\section{OCT features}

OCT features are summarized in Table 2. Preoperatively, the CFT in 21 patients with OCT imaging was $699.8 \pm$ $356.7 \mu \mathrm{m}$, with 11 found to exhibit greater than 1,200 $\mu \mathrm{m}$ of CFT due to submacular hemorrhage. At 1 month after surgery, the thickness was reduced to $432.1 \pm 282.0 \mu \mathrm{m}$, while, at 2 years, it was reduced further to $408.9 \pm 271.6$ $\mu \mathrm{m}$. When assessing CFT values at 24 months after surgery, there were significant changes as compared with both preoperation $(p=0.036)$ and 1-month postoperation ( $p=$ 0.038). However, the CFT at 1-month postoperation was not significantly reduced relative to that recorded preoperatively ( $p=0.067$ ). Additionally, the presence of subretinal hemorrhages was significantly reduced at 2 years after surgery when compared with just 1 month after surgery ( $p$ $=0.001)$ and before surgery $(p<0.001)$. Subfoveal disci- form scarring was detected in $76.9 \%$ of patients by 2 years postoperation.

\section{Predictive factors for long-term visual outcomes}

Predictive factors for a 2-year favorable VA are summarized in Table 3. Univariate analysis revealed that older age (odds ratio $[\mathrm{OR}], 0.879 ; p=0.007$ ) and the presence of submacular hemorrhage (OR, $0.081 ; p=0.022)$ were factors associated with a poor 2-year visual outcome. Subgroup analysis revealed that neovascular AMD showed worse visual prognosis at long-term follow-up relative to in the PCV group, although this was not statistically significant (OR, 0.344; $p=0.078$ ). The trend of worse visual improvement for neovascular AMD as compared with PCV can be appreciated visually in Fig. 1.

For surgical factors, combined cataract surgery $(p=$ $0.518)$ and adjuvant intravitreal anti-VEGF injection ( $p=$ 0.518 ) demonstrated no significant effect on the visual prognosis. The use of several different tamponades, especially for eyes with submacular hemorrhage, was also assessed, including gas ( $p=0.124)$, silicone oil $(p=0.773)$, and tPA $(p=0.418)$, with all showing no correlation with the long-term visual outcome.

Multivariable analysis indicated that older age (OR, 0.876; $p=0.026$ ) and neovascular AMD (as compared with $\mathrm{PCV}$ ) (OR, $0.137 ; p=0.014$ ) were significant negative factors influencing the 2-year visual outcome (Table 3).

\section{Discussion}

In our study, we reported the long-term results of vitrectomy in patients with breakthrough vitreous hemorrhage 
Table 3. Regression analysis of prognostic factors associated with favorable postoperative 2-year visual acuity

\begin{tabular}{|c|c|c|c|c|}
\hline \multirow{2}{*}{ Factor } & \multicolumn{2}{|c|}{ Univariate } & \multicolumn{2}{|l|}{ Multiple $^{*}$} \\
\hline & OR $(95 \% \mathrm{CI})$ & $p$-value & OR $(95 \% \mathrm{CI})$ & $p$-value \\
\hline Age & $0.879(0.801-0.965)$ & $0.007^{*}$ & $0.876(0.780-0.984)$ & $0.026^{*}$ \\
\hline Sex & $1.745(0.560-5.443)$ & 0.56 & - & - \\
\hline Diabetes & $1.810(0.396-8.261)$ & 0.44 & - & - \\
\hline Hypertension & $1.806(0.578-5.643)$ & 0.309 & - & - \\
\hline Preoperative visual acuity & $0.215(0.021-2.251)$ & 0.20 & - & - \\
\hline Presence of massive submacular hemorrhage & $0.081(0.009-0.697)$ & $0.022^{*}$ & $0.118(0.011-1.271)$ & 0.078 \\
\hline \multicolumn{5}{|l|}{ Wet AMD type } \\
\hline PCV & Reference & - & - & - \\
\hline Neo-vascular AMD & $0.344(0.105-1.128)$ & 0.078 & $0.137(0.028-0.665)$ & $0.014^{*}$ \\
\hline \multicolumn{5}{|l|}{ Tamponade type } \\
\hline Gas (SF6 or C3F8) & $6.182(0.608-62.83)$ & 0.124 & - & - \\
\hline Silicone oil & $0.773(0.12-4.96)$ & 0.773 & - & - \\
\hline Combined cataract surgery & $2.60(0.220-30.745)$ & 0.448 & - & - \\
\hline Intravitreal tPA & $0.418(0.25-27.19)$ & 0.418 & - & - \\
\hline Intravitreal anti-VEGF & $2.00(0.629-6.361)$ & 0.240 & - & - \\
\hline
\end{tabular}

Favorable visual acuity: better than finger count $>0.01$; Univariate and multivariate binary logistic regression analysis - only factors thought to be associated with favorable postoperative visual acuity in univariate analysis were included in the multivariate logistic regression model; Each tamponade was compared with the reference value of no tamponade for regression analysis.

$\mathrm{OR}=$ odds ratio; $\mathrm{CI}=$ confidence interval; $\mathrm{AMD}=$ age-related macular degeneration; $\mathrm{PCV}=$ polypoidal choroidal vasculopathy; $\mathrm{tPA}=$ tissue plasminogen activator; VEGF $=$ vascular endothelial growth factor.

${ }^{*}$ Statistically significant value $(p$-value $<0.05)$.

caused by eAMD and identified important prognostic factors influencing the final visual outcome. VA improved significantly and was maintained even at 2 years after surgery. We also found that older age and neovascular AMD type as opposed to PCV type were associated with poorer 2-year visual outcomes.

Previous studies have reported sustained improvement after vitrectomy for eAMD with vitreous hemorrhage. Hasegawa et al. [11] discussed the effectiveness of surgical intervention in a case series with 31 patients followed up with for an average of $19.9 \pm 14.2$ months. Similar to in our study, these authors also observed that the VA recovered to a level similar to the vision at diagnosis or prior to the appearance of vitreous hemorrhage. Our results additionally showed that the 1-month postoperation BCVA stayed quite stable up until 2 years afterward, suggesting that the final visual outcome may be predicted with some accuracy as early as 1 month after surgery. However, in the aforementioned study, unlike in this present study, no significant prognostic factors were identified.

The normal aging process involves many vascular changes to the retina and choroid, leading to reduced choroidal thickness and reduced choroidal flow and perfusion together with structural changes, such as a senescence of the retinal pigment epithelium cells and thickening of Bruch's membrane from the accumulation of waste products and drusen $[12,13]$. These changes, in addition to genetic predisposition and environmental stressors, may be implicated in the pathogenesis of eAMD [14]. Our study also found that age was a strong prognostic factor for predicting surgical outcomes, suggesting that this may be a consequence of age-related retinal degeneration and the 
reduced ability to recover to a previous state.

In our study, we found that the presence of massive submacular hemorrhage, defined as greater than 4-disc diameters in the greatest linear length, was a significant negative factor (OR, $0.081 ; p=0.02$ ) for predicting favorable long-term visual outcomes during univariable regression analysis. This is in line with the established literature: Chang et al. [15] reported that eyes with submacular hemorrhages had worse BCVA outcomes at six months of follow-up, while Cho et al. [16] observed that eyes with PCV developing massive submacular hemorrhages appeared to experience severe vision loss. Thus, the possibility of a massive submacular hemorrhage should be taken into account when performing vitrectomy and patient expectations should be moderated in light of the possibility of a poor visual outcome. Retinal toxicity from the iron contained in degenerating red blood cells and hypoxic damage from reduced nutrient flux may induce long-term postoperative changes including retinal fibrosis and disciform scarring, reducing the final vision [17].

We additionally assessed the impact of adjuvant surgical techniques during vitrectomy, including combining cataract surgery and various combinations of tamponade and intravitreal injections. In terms of combining cataract surgery, previously published studies suggest conflicting results: Roufail et al. [5] observed in five surgeries that combining cataract surgery for the treatment of AMD with breakthrough hemorrhage resulted in poor final outcomes; Chaudhry et al. [18], on the other hand, noted from among six surgeries that combining phacoemulsification appeared to be beneficial to the visual outcome. In our study, combined cataract surgery was not associated with the final visual outcome (OR, 1.45; $p=0.518)$. A subset analysis of the 42 cases with combined cataract surgery in our series found statistically significant vision improvements from preoperative vision ( $p=0.003$ ). Finally, the choice of tamponade, whether gas, silicone oil, or no tamponade, was not found to be an influential factor predicting the final visual outcome.

Numerous studies, including the pivotal ANCHOR and MARINA trials, have emphasized the effectiveness of intravitreal anti-VEGF therapy in eAMD patients [19,20]. In our study, we observed that the average time interval between injections significantly increased after vitrectomy to more than 20 months after surgery. This resulted in the reduction of the average number of injections per patient performed after vitrectomy. The prolonged stabilization and reduced disease activity after vitrectomy noted in this study may be the result of increased oxygenation saturation in vitrectomized eyes [21] and reduced the oxygen need as a result of retinal atrophy and reduced metabolic activity. In fact, Baek et al. [22] observed similarly diminished disease activity in PCV eyes after the occurrence of submacular hemorrhage and postulated that the reduced number of polyps seen following hemorrhage occurrence may be the primary cause. Their study showed that, in 52 PCV eyes, at least one polypoidal lesion disappeared after hemorrhage in seven of 10 eyes $(70 \%)$ and the injection frequency diminished after hemorrhage as compared with in control eyes, which were PCV eyes without hemorrhage $(p<0.001)$ [22].

Finally, in line with the current literature [23], we also observed that PCV eyes had relatively more favorable visual outcomes than did neovascular AMD eyes. We compared the VA between the two subtypes of eAMD and a significant difference was noted in vision at 1-year postoperation. However, further analysis did reveal that this difference appeared to lose statistical significance by the second year. Further long-term studies might reveal the ultimate prognosis of eAMD patients depending on these subtypes.

This was a retrospective study with limitations inherent to its design, without control of the baseline characteristics and heterogeneity of the data in terms of adjuvant surgical techniques. Also, vitrectomy was performed by several different surgeons. We were able to find an association between surgical treatment and decreasing anti-VEGF treatment intervals/numbers; however, further clinical studies focusing on anti-VEGF and vitrectomy are required to establish the possibility of a causal relationship. The strengths of our study are that we included a relatively large number of patients with long-term follow-up and our findings encompassed results from both PCV and neovascular AMD eyes. Future carefully designed prospective studies will help to elucidate the optimal combination of adjuvant techniques to achieve the best visual outcomes.

In conclusion, we report the long-term visual outcomes and possible prognostic factors for patients with vitreous hemorrhage associated with eAMD undergoing surgical intervention. Age at onset and differentiating PCV with typical neovascular AMD appear to be important prognostic factors predicting the long-term visual outcomes. Alto- 
gether, vitrectomy resulted in visual improvement. Breakthrough vitreous hemorrhage in eAMD appears to be associated with a decreased need for anti-VEGF therapy.

\section{Conflict of Interest}

No potential conflict of interest relevant to this article was reported.

\section{Acknowledgements}

This study was supported by a faculty research grant from the Yonsei University College of Medicine (6-20160113).

\section{References}

1. Wong WL, Su X, Li X, et al. Global prevalence of age-related macular degeneration and disease burden projection for 2020 and 2040: a systematic review and meta-analysis. Lancet Glob Health 2014;2:e106-16.

2. Congdon N, O'Colmain B, Klaver CC, et al. Causes and prevalence of visual impairment among adults in the United States. Arch Ophthalmol 2004;122:477-85.

3. Googe JM, Hirose T, Apple DJ, Melgen S. Vitreous hemorrhage secondary to age-related macular degeneration. Surv Ophthalmol 1987;32:123-30.

4. Azzolini C, Menchini U, Pece A, et al. Age-related macular degeneration and vitreous hemorrhage. Eur J Ophthalmol 1991;1:142-7.

5. Roufail E, Polkinghorne PJ. Combined cataract surgery and vitrectomy for vitreous haemorrhage secondary to age-related macular degeneration. Clin Exp Ophthalmol 2008;36:36-8.

6. Kang HG, Kang H, Byeon SH, et al. Long-term visual outcomes for treatment of submacular haemorrhage secondary to polypoidal choroidal vasculopathy. Clin Exp Ophthalmol 2018;46:916-25.

7. Li ZX, Hu YJ, Atik A, et al. Long-term observation of vitrectomy without subretinal hemorrhage management for massive vitreous hemorrhage secondary to polypoidal choroidal vasculopathy. Int J Ophthalmol 2019;12:1859-64.

8. Lin HC, Yang CH, Yang CM; Medscape. Visual outcomes of vitrectomy for polypoidal choroidal vasculopathy-related breakthrough vitreous haemorrhage. Eye (Lond) 2014;28:797-806.

9. Choi EY, Han JY, Lee H, et al. Impact of antibiotic resistance of pathogens and early vitrectomy on the prognosis of infectious endophthalmitis: a 10-year retrospective study. Graefes Arch Clin Exp Ophthalmol 2019;257:805-13.

10. Muda R, Vayavari V, Subbiah D, et al. Endogenous endophthalmitis: a 9-year retrospective study at a tertiary referral hospital in Malaysia. $J$ Ophthalmic Inflamm Infect 2018;8:14

11. Hasegawa T, Otani A, Sasahara M, et al. Prognostic factors of vitreous hemorrhage secondary to exudative age-related macular degeneration. Am J Ophthalmol 2010;149:322-9.

12. Ehrlich R, Harris A, Kheradiya NS, et al. Age-related macular degeneration and the aging eye. Clin Interv Aging 2008;3:473-82.

13. Friedman E. A hemodynamic model of the pathogenesis of age-related macular degeneration. Am J Ophthalmol 1997;124:677-82.

14. Fine SL, Berger JW, Maguire MG, Ho AC. Age-related macular degeneration. $N$ Engl J Med 2000;342:483-92.

15. Chang YS, Kim JH, Kim JW, et al. Development of submacular hemorrhage in neovascular age-related macular degeneration: influence on visual prognosis in a clinical setting. Korean J Ophthalmol 2018;32:361-8.

16. Cho JH, Ryoo NK, Cho KH, et al. Incidence rate of massive submacular hemorrhage and its risk factors in polypoidal choroidal vasculopathy. Am J Ophthalmol 2016;169:7988 .

17. Stanescu-Segall D, Balta F, Jackson TL. Submacular hemorrhage in neovascular age-related macular degeneration: a synthesis of the literature. Surv Ophthalmol 2016;61:18-32.

18. Chaudhry NA, Flynn HW, Murray TG, Belfort A. Combined cataract surgery and vitrectomy for breakthrough vitreous hemorrhage from age-related macular degeneration. Ophthalmic Surg Lasers 2002;33:16-8.

19. American Academy of Ophthalmology. Age-related macular degeneration: preferred practice pattern guideline [Internet]. San Francisco: American Academy of Ophthalmology; 2014 [cited 2020 Feb 7]. Available from: http://www.aao.org/ Assets/db935a77-1997-4d60-b850-71b7602f46e2/63558214 3853270000/age-related-macular-degeneration-ppp-pdf.

20. Agarwal A, Rhoades WR, Hanout M, et al. Management of neovascular age-related macular degeneration: current state-of-the-art care for optimizing visual outcomes and 
TY Kim, et al. Surgical Outcomes for Vitreous Hemorrhage in AMD

therapies in development. Clin Ophthalmol 2015;9:1001-15.

21. Lim LS, Tan L, Perera S. Retinal vessel oxygen saturation increases after vitrectomy. Invest Ophthalmol Vis Sci 2014;55:3851-6.

22. Baek J, Kim JH, Lee MY, Lee WK. Disease activity after development of large subretinal hemorrhage in polypoidal choroidal vasculopathy. Retina 2018;38:1993-2000.

23. Wong CW, Yanagi Y, Lee WK, et al. Age-related macular degeneration and polypoidal choroidal vasculopathy in Asians. Prog Retin Eye Res 2016;53:107-39. 